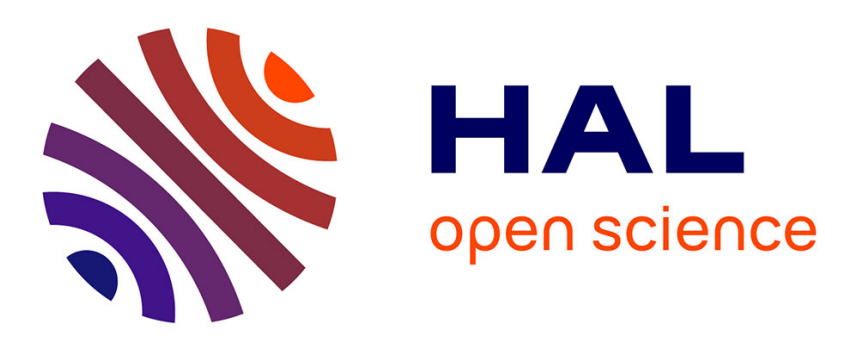

\title{
Iconographie et liturgie dans les études médiévales aujourd'hui : un éclairage méthodologique
}

\author{
Eric Palazzo
}

\section{To cite this version:}

Eric Palazzo. Iconographie et liturgie dans les études médiévales aujourd'hui: un éclairage méthodologique. Cahiers de Civilisation Médiévale, 1998, 41 (161), pp.65-69. 10.3406/ccmed.1998.2711. halshs-01341176

\section{HAL Id: halshs-01341176 \\ https://shs.hal.science/halshs-01341176}

Submitted on 4 Jul 2016

HAL is a multi-disciplinary open access archive for the deposit and dissemination of scientific research documents, whether they are published or not. The documents may come from teaching and research institutions in France or abroad, or from public or private research centers.
L'archive ouverte pluridisciplinaire HAL, est destinée au dépôt et à la diffusion de documents scientifiques de niveau recherche, publiés ou non, émanant des établissements d'enseignement et de recherche français ou étrangers, des laboratoires publics ou privés. 


\section{Iconographie et liturgie dans les éludes médiévales aujourd'hui : un éclairage méthodologique}

Éric Palazzo

\section{Citer ce document / Cite this document :}

Palazzo Éric. Iconographie et liturgie dans les éludes médiévales aujourd'hui : un éclairage méthodologique. In: Cahiers de civilisation médiévale, 41e année ( $\left.n^{\circ} 161\right)$, Janvier-mars 1998. pp. 65-69;

doi : 10.3406/ccmed.1998.2711

http://www.persee.fr/doc/ccmed_0007-9731_1998_num_41_161_2711

Document généré le 01/06/2016 


\section{Iconographie et liturgie dans les études médiévales aujourd'hui : un éclairage méthodologique}

En prévision de la parution du quarantième volume des Cahiers de civilisation médiévale. la direction de la revue a eu l'excellente idée d’engager une réflexion sur les recherches actuelles des médiévistes. Ceci s’est principalement concrétisé avec la publication d’articles émanant de la plume de médiévistes de renom. regroupés dans un des numéros de l'année 1996'. Cette réflexion à plusieurs voix. très stimulante, invite tout médiéviste à se pencher sur son propre travail, à le scruter d'un oil critique et, surtout, à examiner sa place dans la polyphonie que représentent les études médiévales. C'est dans ce but qu'ont été écrites les lignes qui suivent. Elles ont pour simple objectif de mettre en lumière les enjeux majeurs - du moins à mes yeux - des recherches actuclles portant sur les rapports entre iconographic et liturgic.

Afin d'orienter mes réflexions, je suis parti des lignes directrices données par Piotr Skubiszewski dans les pages introductives qui ouvrent le recucil darticles auquel jai fait allusion plus haut ${ }^{2}$. Dans le texte. jai notamment été frappé par l'insistance justifiéc de l'auteur sur le bon choix opéré par le médiéviste en ce qui concerne ses sujets dinvestigation. Or ceux-ci. outre l’influence inévitable de l'environnement idéologique du chercheur. sont de plus en plus déterminés par les nécessités du travail pluridisciplinaire ou interdisciplinaire (je reviendrai plus loin sur la distinction à établir entre ces deux qualificatifs). Mais le travail pluridisciplinaire du médiéviste ne doit en aucun cas signifier la disparition progressive de la spécificité de chaque discipline. La méthodologie et les finalités propres à chacune d'elles doivent rester la règle et j’ajouterai même la condition sine qua non pour la réalisation d'une pluridisciplinarité efficace et fructueuse. Dans ce sens. nous faisons nôtre l'idéc du va-et-vient permanent d’une discipline à l'autre par ceux qui pratiquent intelligemment la pluridisciplinarité, guidés sans cesse par le souci d'une démarche conceptuelle. présente en arrière-plan.

À propos de ces différents points, que peut-on dire des recherches mêlant liiconographie et la liturgie? Avant de proposer quelques réponses et suggestions, je rappellerai que de nombreux autcurs ont contribué à faire progresser cette réflexion. Je ne peux, dans le cadre de cette note. faire le point sur l'historiographie du sujet. ce qui mériterait un article en soi. Le lecteur sera ainsi peut-être déçu de ne pas trouver les références bibliographiques à telle étude essentielle pour le propos. Ceci étant. dans le cours de mes réflexions. je signalerai à l'occasion certaines études qui ont marqué les recherches sur l'iconographie et la liturgie, ou bien encore, je mentionnerai les différences dapproche du sujet d'une tradition historiographique à l'autre. Je ne prétends pas non plus traiter ici du domaine beaucoup plus large encore de létude des images par les médiévistes d’horizons divers ${ }^{3}$. Je constate cependant la rencontre de problèmes souvent identiques entre les études sur "iconographie et histoire médiévale " el "iconographic et liturgie ».

Dans un premier temps. jexposerai rapidement les positions respectives des historiens de lart et des liturgistes lorsqu ils abordent des sujets de recherche où iconographie et liturgie se rencontrent. Ce parti. on s'en doute. mettra en relief les défauts des uns et des autres, à l'origine d'une pluridisciplinarité malmenée.

Dans un second temps, je ferai un certain nombre de propositions de nature méthodologique, visant à donner, jose l'espérer, plus defficacité à la rencontre entre iconographic et liturgie.

1. Cahiers de civilisation médiévale, 39. 1996. facs. 1-2.

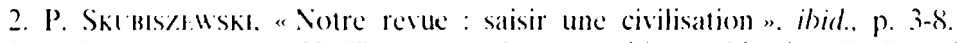

3. Voir entre autres. H. Tol BI:RI. "Iconographic et histoire de la spiritualité médiévale ". Rélae dhistorire de la spirimulité, 50. 1974. p. 265-284 (texte repris dans Cin art dirigé. Réforme gregorienne et icomegraphie. Paris, 1990. p. 19-36). ou bien encore J.-( 1 . S(nsils. "I.a culture de limago". Amnales IISS. 1996/1. p. 3-.36. 


\section{Les historiens de l'art et les liturgistes face à l'interdisciplinarité.}

D’emblée, je propose d'échanger le terme de pluridisciplinarité par celui d'interdisciplinarité. La raison essentielle de ce choix sémantique réside dans le sens même de chacun des mots. En effet. tandis que la pluridisciplinarité implique un mélange - même fructueux - des disciplines. l'interdisciplinarité suppose. elle. une combinaison harmonieuse de ces dernières. À mes yeux. le risque de la pluridisciplinarité tient à la confusion des genres, à un mélange des disciplines sans véritable réflexion sur les objectifs à atteindre. Au contraire. l'interdisciplinarité me paraît nécessiter au préalable une façon de mêler plusieurs disciplines où chacune d'elles ne serait utilisée qu à bon escient. juste pour ce qu elle peut apporter à la compréhension d'un objet de recherche.

Cette précision terminologique étant faite. passons maintenant aux positions respectives des historiens de l'art et des liturgistes sur l'interdisciplinarité. Parmi les historiens de l’art. ceux qui pratiquent les recherches en iconographie savent bien toutes les difficultés inhérentes à ce genre de démarche. Sans mattarder ici sur la place de l'iconographie dans les travaux d'historiens de l'art, je veux plutôt insister sur ce qui me paraît être le défaut essentiel des iconographes face à la liturgie : le déterminisme fonctionnel ou la notion de fonctionnalité. En effet. cherchant visiblement à acquérir leurs lettres de noblesse auprès d’autres catégories de chercheurs. les historiens de l'art. spécialistes de l'iconographic. ont de façon générale tendance à ne voir les rapports entre iconographie et liturgie que du point de vue de la fonctionnalité des images. Héritée à la fois des traditions du XIX ${ }^{\mathbb{C}} \mathrm{s}$. comme de celles plus contemporaines où tout doit s'expliquer par une fonction. l'explication fonctionnelle de l'iconographie. en rapport avec la liturgie. étouffe la réalité des liens entre les images et le rituel. Par exemple. lorsque des énigmes iconographiques surgissent à la vue de l'historien de l'art - principalement à propos des programmes monumentaux peints ou sculptés dans les églises - celui-ci a la fâcheuse tendance à considérer que le "mystère " de l'iconographie doit s"expliquer par ses liens avec la liturgie ${ }^{4}$. Celle-ci, prise alors dans son acception la plus large, voire la plus mal définie. sert en quelque sorte. à ce moment de la démarche. de solution "miracle" qui permettrait de résoudre toutes les énigmes iconographiques léguées par le moyen âge. À cela. vient sajouter le défaut de la recherche de fonctionnalité à outrance. Dans ce contexte, le discours tient en ces quelques mots : l'image. avec ses curiosités iconographiques. est là pour servir une fonction rituelle. Récemment encore, participant à un colloque international organisé à Rome par la Biblioteca Hertziana sur le thème : "Arte e liturgia nel medioevo". j’ai été frappé de constater que la majeure partie des intervenants se situait clairement dans cette optique de la recherche de la fonctionnalité de lïmage dans la liturgie. Tout cela sans proposer pour autant une réflexion sur la nature des liens entre iconographie et liturgie. et en acceptant comme allant de soi lidée de la fonctionnalité. Or. comme on le verra plus loin. la raison dêtre de l'image au moyen âge - quel que soit son support - ne réside pas uniquement dans sa fonctionnalité rituelle, mais parfois aussi dans ce qu'elle vaut pour elle-même, sans lien direct avec une fonction liturgique précise.

De leur côté, les liturgistes, pour des raisons quiil serait trop long de développer ici. ont, dans l'ensemble. une vision démesurément "théologique» de l'image: je m'explique. Aux yeux de la majeure partie des spécialistes de la liturgie médiévale. l'image - et a fortiori l'iconographie - n’a pas véritablement de valeur en soi. Elle n’est pour ainsi dire là que pour soutenir l'expression d’une théologie de la liturgie. Dans ce sens. l'iconographie apparaît forcément comme secondaire en comparaison du message théologique du rituel. el elle ne contribue pas à préciser ou à moduler ce message. Ainsi. les images sont trop souvent considérées par les liturgistes comme de belles illustrations du rituel ou d'une idée théologique. Elles sont là pour "faire beau", parce que la liturgie doit comporter des éléments esthétiquement repérables. Ceci n’est en soi pas faux - du moins dans la conception médiévale de l'esthétique liturgique - mais offre malheureusement la possibilité d'une réduction de l'image et de son iconographie à un simple decortum visuel pour agrémenter te licu ou le moment de la liturgie.

Cette vision excessivement théologique de l'image dans la liturgie a également pour conséquence denfermer liconographic dans une sorte de carcan spirituel - pris au sens médiéval du terme. Ainsi, les images réalisées dans l'espace du culte ou sur des objets destinés à servir dans ce cadre. obéiraient

4. Jai développé ces questions dans Les sacramentaires de Fulda. Étude sur l'iconographice "la liturgice à l'ápongue ottonienne. Münster. 1994 (1.iturgiewissenschaftliche Quellen und Forschungen. 77). p. 159-164. 
forcément à la subtilité du discours de la théologie de la liturgie ${ }^{5}$. Bien sûr. j’aurai l'occasion plus loin de préciser ee point. l'image liturgique présente bien des liens avec telle ou telle idée de la théologie de la liturgie. mais elle n'en est pas pour autant une illustration directe. "asservie " par la hauteur du discours théologique ou bien encore par les textes liturgiques. En réalité. les rapports entre iconographie et textes théologiques ou sacrés présentent bien plus de subtilité quiil n’en paraît au premier regard.

\section{Iconographie et liturgie : propositions méthodologiques}

Les propositions méthodologiques concernant les rapports entre iconographie et liturgie sont relatives à différents aspects de la problématique d'ensemble. Tout d'abord. je souhaite ici mettre l'accent sur l'impérative nécessité de distinguer plusieurs niveaux de lecture et de compréhension d'une image dans ses rapports avec la liturgie. Bien souvent. une analyse minutieuse de liconographie d'un programme monumental. d'une image dans un manuscrit liturgique. ou bien encore de thèmes gravés ou sculptés sur un objet destiné au culte. révèle la coexistence de plusieurs niveaux d'interprétation de l'image en rapport avec la liturgic. En effet. ceux-ci peuvent aussi bien concerner la proximité d'un sujet iconographique avec la thématique globale d'un texte liturgique ou de la théologie d'une fête. qu un lien plus ou moins immédiat entre lïmage ou un ensemble d'images avec le lieu de la célébration. Autrement dit. rares sont les cas où. dans un contexte liturgique global. la compréhension de l'iconographie ne se ferait quà partir d'un seul niveau d'interprétation.

Ensuite. d’un type de support à l'autre, la fonctionnalité d’une image dans le contexte de la liturgie ne s'établit pas d'une façon automatique et systématique. Par exemple. les images monumentales placées sur les parois des églises ne se comprennent pas obligatoirement dans un simple rapport de fonctionnalité au moment du déroulement du culte. Les images liturgiques, prises au sens large, n'ont en effet pas été spécialement conçues pour être vites. Au moyen âge. le lien entre lïconographic et la liturgie passe également par la notion de présence. L'essentiel aux yeux des contemporains était que l'image - quel que soit le support - soit là, présente au moment du déroulement de la liturgie. Dans ce sens, on peut considérer la fonction de limage dans la liturgie en terme de relation intellectuelle. conceptuelle. avec le thème d'une messe, avec les textes lus, chantés ou priés à cette occasion. Ici. l'image participe, au même titre que l'ensemble rituel déployé pour le déroulement d'une cérémonie particulière. à lexistence de la liturgie, à sa raison dêtre. À lappui de cette manière de concevoir les rapports entre iconographie et liturgie. il faut rappeler une évidence qui semble bien trop souvent laissée soigneusement de côté par les historiens de l'art et les liturgistes. En effet. les conditions du déroulement des cérémonies nous échappent en grande partie. Mais on peut facilement concevoir le déroulement d"une messe, d'un office ou d'une autre cérémonic particulière - par exemple l'exécution de la vigile pascale - qui impliquait bien souvent que les acteurs - sans parler des spectateurs - ne voyaient pas les images. Mais léssentiel était bien que ces dernières aient été là afin dajouter, par leur présence. "quelque chose" au déroulement de la liturgie. en lien avec le contenu de l'iconographie et son sens souvent complexe. Dans ce contexte. on šéloigne rapidement d'une fonctionnalité simple et directe quaffectionnent les hommes du $\mathrm{xx}^{\mathrm{c}} \mathrm{s}$. Ceci ne veut pas pour autant définitivement exclure de la conception médiévale un lien fonctionnel entre les images et la liturgie. Je crois cependant à la relative rareté de ce lien et. en sens inverse. à lexpression d'une fonctionnalité moins immédiate des images au moment du déroulement de la liturgie. mais plus globale. prenant en considération la notion médiévale de presentia.

Continuons l'énoncé de ces propositions méthodogiques en revenant sur les différents niveaux d'interprétation afin d'en préciser le sens et le contenu. Comme je l'ai dit plus haut. liconographie et la liturgie n'entretiennent pas des rapports de dépendance qui consisteraient. par exemple. à enfermer une image dans une lecture théologique. Les images font partie intégrante du rituel. au même titre que les textes sacrés. le lieu de la célébration. les vêtements et les objets liturgiques. et bien dautres choses encore. Ainsi. dans le contexte de la liturgie. liconographie ne peut se comprendre si on lisole du contexte global que forme le rituel. Par ailleurs. il faut rappeler les règles propres de liconographie qui ne correspondent pas toujours à la volonté d'exprimer une iclée théologique ou bien à mettre en image

5. Voir. par exemple. les positions déja anciennes mais néanmoins toujours dactualité prises par le grand liturgiste

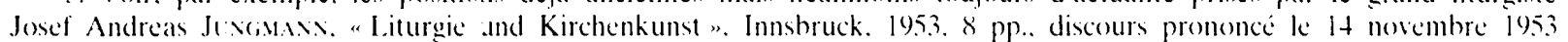
a l'niversite d'Innshruck a l'occasion de laceession au rectorat de Jungmann. 
le contenu d'un texte liturgique. Ajoutons encore un point rarement pris en compte : certaines images "liturgiques", je pense en particulier aux peintures monumentales ou aux sculptures des églises. ne sont considérées comme telles qu'une partie du temps, c'est-à-dire celui du déroulement des célébrations. Or, comme aujourd hui. une église au moyen âge nećtait pas en permanence le théâtre des diverses cérémonies de la liturgie. Pendant la journée. selon le type d'église. d’autres affectations étaient réservées à ces lieux et l'image peinte ou sculptée était - si jose dire - laissée au repos pendant un certain temps.

À propos des différents niveaux d'interprétation d'une image dans le contexte de la liturgie. je dirais que l'on a affaire à une sorte de jeu subtil et d'équilibre entre les traditions propres à l'íconographic et le contenu d'un rite ou d'une fête liturgique. En effet. il faut parfois aller chercher bien loin l'influence d'une oraison ou d'un commentaire exégétique sur telle péricope biblique pour comprendre un simple motif iconographique contenu dans une image où le thème et la façon de le traiter apparaissent a priori fort traditionnels. Cette remarque implique de la part de l'historien de l'art une attention sans relâche envers les textes liturgiques, dans leur grande diversité, ainsi que par rapport à la connaissance de l'histoire des rites à travers l'espace et le temps" ${ }^{6}$. Autrement dit. sans se métamorphoser en spécialiste de la liturgie et de son histoire. la connaissance suffisante du contenu des textes, de leurs traditions, des pratiques rituelles et de leur évolution, doit permettre à l'historien de l'art soucieux d'interdisciplinarité de situer plus précisément la dimension "liturgique " d'une image et de son iconographie. Par ailleurs. en ce qui concerne les liturgistes, il est grand temps qu ils cessent de considérer l'image comme une simple illustration de complexes idées théologiques ou exégétiques véhiculées par la liturgie - ce qui peut, je le répète, parfois être le cas ${ }^{7}$. Un minimum de connaissance par les liturgistes de l'iconographie et de la subtilité de son discours serait grandement souhaitable afin de leur permettre d'échapper à une vision trop unilatérale des rapports entre iconographie et liturgie. Les liturgistes prêts à briser les barrières des disciplines mettraient encore à profit cette connaissance pour saisir un élément fondamental du rituel : sa dimension visuelle. En effet, la liturgie ne se limite pas à des textes, à des gestes, ou à des acteurs. Elle intègre, de façon complémentaire et cohérente. à tous ces éléments. une dimension spatiale et visuelle où les images occupent une place centrale.

Un autre aspect me paraissant relever de l'approche méthodologique concernant les rapports entre iconographie et liturgie touche à la diversification croissante du concept même de liturgie. En effet. depuis plusieurs années, on assiste à la prise en compte par les historiens notamment. mais également par certains historiens de l'art, des manifestations relatives aux pratiques dévotionnelles face à l'image ${ }^{8}$. Grâce à l'étude croisée de textes fort divers - souvent il s’agit de textes hagiographiques - et d'images de type dévotionnel. un pan nouveau des rapports entre iconographie et rituel se découvre progressivement à nos yeux. Évidemment, cette approche originale implique l'élargissement de la notion même de liturgie, entendue alors non plus seulement comme la manifestation rituclle de l'Église officielle mais comprenant aussi des pratiques plus ou moins codifiées ou encadrées par les autorités ecclésiastiques, où l'image intervient de façon particulièrement forte. Dans ce cadre, les rapports entre iconographie et liturgie se manifestent de façon souvent plus subtile que dans des contextes rituels et artistiques bien déterminés et font intervenir plus en profondeur la perception de limage par

6. Voir. par exemple. l'étude exemplaire menée par O. K. Wi:kckmisIter sur le fragment de linteau de l'ancien portail nord de la cathédrale d’Autun. représentant Ėve. "The Lintel Fragment Representing Eve from S. Lazare. Autun". Journal of the Warburg and Comrtauld Institutes, 35, 1972. p. 1-30. Cet A. a tenté - selon moi avec moins de succies une approche interdisciplinaire analogue à celle menée à propos du linteau d’Eve. appliquée cette fois au portail nord de la cathédrale d’Autun. représentant le Jugement dernier. "Die Auferstehung der Toten am Westportal von S. Lazare in Autun ". Frilhmittelallerliche Studien. 16. 1982. p. 208-236.

7. Voir, par exemple, les remarquables études du liturgiste américain R. E. Rexvoloss. "Image and Text : A (arolingian Illustration of Modifications in the Early Roman Eucharistic Ordines". Viator. 14, 1983, p. 59.75 et "Image and Text : The Liturgy of Clerical Ordination in Early Medieval Art ". (jesta. XXII. 1983. p. 27-38.

8. Voir le livre fort stimulant et parfois à juste titre controversé de Hans BHolscig. Bild und Kult. Eime (jeschichte des Bildes vor de'm Zeitalter der Kunst. Munich. 1990). Voir aussi. à titres dexemples. A. ('astes. "La dévotion privée et lart à lépoque carolingienne : le cas de Sainte-Maure de Troyes". Cahiers de civilisation médiévale. 33. 1990). p. 3-18 (bien que le texte utilisé par latuteur comporte encore de nombreuses incertitudes quant à sa date). Jai fait le point sur une partic de ees questions relatives a la pratique dévotionnelle el aux images. "I es pratiques liturgiques el dévotionnelles et le décor monumental dans les églises du Moyen Âge". dans L:Emplacement et la fonction des images dams la peinture murale du Moyen Âge. Actes du V' séminaire international d'art mural. Saint-Sarin. 1992. Saint-Sasin. 1993. p. 45-56. 
l'acteur ou le spectateur dans l'exécution d’un acte dévotionnel. celui-ci étant placé en marge de la liturgie officielle.

Je terminerai enfin ces quelques réflexions par le souhait de voir des recherches nouvelles sur l'iconographie et la liturgie se développer mais sans jamais perdre de vue leur dimension historique. En effet, l'affinement des méthodes d’investigation visent non seulement à rendre plus fructucuse l'interdisciplinarité - en l'occurrence du point de vue des rapports entre iconographie et liturgie mais aussi à intégrer de façon plus prégnante ces recherches dans l'histoire médiévale au sens large. Comment en effet aujourd hui occulter les apports de l'histoire sociale - pour ne prendre qu un exemple parmi d'autres - dans le domaine conjoint de l'iconographie et de la liturgie"? Les images comme les pratiques rituelles font partic intégrante de la culture du moyen âge. de la civilisation médiévale et. en ce sens. elles ne peuvent être totalement appréhendées, dans leur complexité globale. sans un souci permanent de les voir prendre part au grand concert des études médiévales.

Éric Palazzo)

CNRS/IRHT-Orléans

3. avenue de la Recherche-Scientifique 45071 ORLÉANS Cedex 